\title{
Case report: hypereosinophilic syndrome response to infliximab in a patient with ulcerative colitis
}

\begin{abstract}
Introduction: We present a case of a caucasian 83-year-old female with a previous diagnosis of ulcerative colitis (UC) admitted to our institution with a mild UC flare and a hypereosinophilic overlap syndrome refractory to corticosteroids but that responded to intravenous infliximab.

Conclusion: Hypereosinophilic Syndrome (HES) is a very rare disorder that usually appears in people between 20 to 50 years old although it can also occur in elderly people and children. It affects equally to both sexes and several mechanisms have been proposed related to the excess in the synthesis of eosinophils. The clinical course may be silent or present different manifestations depending on the affected tissue. The diagnostic workup should include blood tests, imaging tests and tissue biopsies. A bone marrow aspiration and biopsy are necessary if all the previous studies are normal. Early diagnosis and treatment of HES have a significant impact on survival. Treatment of asymptomatic patients is based on close monitoring to prevent complications. In symptomatic cases, empiric corticosteroids or other immunosuppressive agents should be used. Hematopoietic cell transplantation is the last therapeutic alternative.
\end{abstract}

Keywords: Hypereosinophilic Syndrome (HES), Ulcerative Colitis, Infliximab, hypereosinophilia
Volume 5 Issue 5 - 2016

\author{
Solano Marina,' Lacarta Pedro,' Ferrández \\ Ángel, ${ }^{1,2}$ Gomollón Fernando, ${ }^{1,2}$ Lanas \\ Ángel 1,2 \\ 'Department of Digestive Diseases, Hospital Clínico \\ Universitario Lozano Blesa, Spain \\ ${ }^{2}$ CIBERehd, Spain
}

\begin{abstract}
Correspondence: Ferrández Angel, Department of Digestive Diseases, Hospital Clínico Universitario Lozano Blesa, Zaragoza. San Juan Bosco n 15, 50009 Zaragoza, Spain, Tel 976765700 ,
\end{abstract} Email angel.ferrandez@telefonica.net

Received: September II, 2016 | Published: November 28, 2016

\section{Introduction}

There are many conditions associated with plasma and tissue eosinophilia (Table 1). The degree of eosinophilia can be mild $(500-1500$ Eos $/ \mathrm{mL})$, moderate $(1500-5000 \mathrm{Eos} / \mathrm{mL})$ or severe ( $>5000$ Eos $/ \mathrm{mL}$ ). Hypereosinophilic Syndrome (HES) is defined as either hypereosinophilia over 1500 eosinophils $/ \mathrm{mL}$ in at least 2 determinations or as tissue damage secondary to eosinophilic infiltration $\cdot 1,2$. The present case report is a patient with a previous diagnosis of ulcerative colitis (UC) admitted to our institution with a suspected mild UC flare and a hypereosinophilic overlap syndrome refractory to corticosteroids but who responded to infliximab.

Table I Pathologies associated with eosinophilia

Allergies
Infections
Lynphoproliferative disorders
Neoplasms
Organ specific diseases
Immunological
Endocrine

\section{Case report}

A caucasian 83-year-old female with no drug allergies and with a previous history of extensive UC diagnosed 2 years before being admitted to our hospital on September 8th 2013 due to bloody diarrhea, with 18-20 passes per day during the last month. She also complained of abdominal pain, hyporexia and weight loss. She was hemodynamically stable, with mild signs of dehydration. Physical examination showed diffuse abdominal pain but no signs of peritoneal irritation and peristalsis was preserved. She presented edema with fovea in both lower limbs extended to both knees. Digital rectal examination did not reveal masses.
Laboratory tests showed inflammatory activity with erythrocyte sedimentation rate (ESR) $50 \mathrm{~mm}, \mathrm{C}$ reactive protein (CRP) $2.52 \mathrm{mg} /$ $\mathrm{dL}, 28500$ leukocytes/mm3 (neutrophils 17000/mm3, eosinophils $8900 / \mathrm{mm} 3$ ), $445000 / \mathrm{mm} 3$ platelets, hemoglobin $12.3 \mathrm{~g} / \mathrm{dL}$, blood iron $19 \mathrm{mg} / \mathrm{dL}$, albumin $1.8 \mathrm{~g} / \mathrm{d}$. According to Truelove criteria, the patient has a severe flare up ( 21 points). She had an abdominal RX done which showed nothing relevant and she was started on intravenous corticosteroids and antibiotics (ciprofloxacin plus metronidazole), oral nutritional and iron supplementation. A colonoscopy was performed and revealed a severe ulcerative colitis. No histological or immunohistochemical signs of super infection by cytomegalovirus (CMV) were observed. Stool cultures and parasites in stool were both negative.

Clinical evolution was good and the patient was discharged from hospital with oral medication and ambulatory monitoring. Two weeks later, on September 26th, the patient was again admitted with a mild UC flare. Laboratory tests showed thrombocytopenia $(77000 \mathrm{mil} /$ $\mathrm{mm} 3)$ and an increased leucocyte $(36,300$ per mm3) and eosinophil $(19,700$ per mm3) count. Eosinophilia was present 5 months before the flare (since May 2013), before corticoid therapy was used, with a steady and significant increase to $31.400 / \mathrm{mm} 3$ (Figure 1). Bone marrow aspiration, gallium scintigraphy and abdominal CT scan were performed to rule out a myeloproliferative syndrome. They were normal and the patient was diagnosed an overlap Hypereosinophilic Syndrome. Clinical evolution was torpid and the patient developed nosocomial pneumonia and urinary tract infection by $E$. Coli and Proteus miriabilis receiving broad-spectrum antibiotic. Once myeloproliferative syndrome was excluded the patient was treated with intravenous Infliximab $(5 \mathrm{mg} / \mathrm{kg})$ achieving clinical and biochemical remission after the second infusion. After one year of infliximab treatment the patient remains in complete remission(Figure $1)$. 


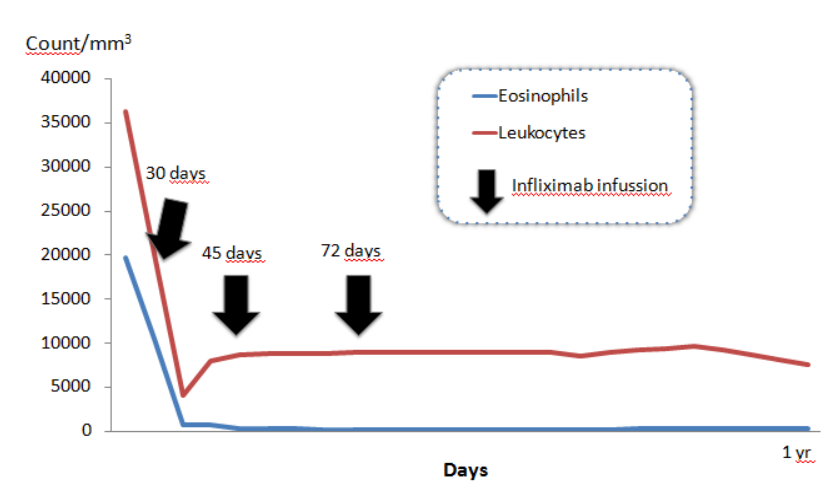

Figure I Infliximab infussion.

\section{Discussion}

Hypereosinophilic Syndrome (HES) is a rare disorder that usually appears in people between 20 to 50 years old but it can also occur in elderly people and children. Both sexes are affected in the same proportion. Several mechanisms have been related to the overproduction of eosinophils:

a. Clonal eosinophil proliferation secondary to a primary genetic defect that alters hematopoiesis stem cell and/or defects in the signal transducing receptors mediators of lymphopoiesis

b. Overproduction of cytokines such as IL-5.

c. Regulatory defects in the suppressor system of eosinophilopoiesis. The World Health Organization established a classification of different molecular subtypes for eosinophilia:

i. Myeloid and lymphoid neoplasms with eosinophilia and abnormalities of PDGFRA, PDGFRB or FGR1,

ii. Unexplained chronic eosinophilic leukemia,

iii. Lymphocytic eosinophilia and

iv. HES, this, being an exclusion diagnosis ${ }^{1,3,4}$.

The clinical course may be silent or presenting dermatological (37\%), pulmonary $(25 \%)$, gastrointestinal $(14 \%)$ or cardiac $(5 \%)$ manifestations. Liver involvement may occur as a chronic active hepatitis, hepatic focal lesions, eosinophilic cholangitis or Budd Chiari syndrome. The diagnostic workup should include blood tests including liver enzymes, creatine kinase, troponins and renal function, electrocardiogram, echocardiogram, chest radiography, chest and abdominal CT, and tissue biopsies. Several biomarkers are currently under study and could be useful as predictors of different subtypes of HES. Serum tryptase, vitamin B12 and serum IgE have to be analyzed. A bone marrow aspiration and biopsy with immunohistochemical and molecular techniques $(1,5)$ are necessary if all the previous studies are normal.

Treatment of asymptomatic patients is based on close monitoring to prevent complications. In symptomatic cases, empiric corticosteroids should be started (which are the first option in lymphoid variants). Other drugs such as Imatinib (for PDGFRA or PDGFRB variants), hydroxyurea or leukapheresis are used in refractory cases to steroids. Treatment with alpha interferon, mepolizumab (anti-IL5), alemtuzumab (anti-CD52) and other chemotherapeutic agents such as cladribine, chlorambucil, vincristine, methotrexate, cyclosporine and etoposide have been evaluated in specific cases. Hematopoietic cell transplantation should be considered as the last therapeutic alternative when everything else has failed ${ }^{1-10}$. Anti-TNF administration has been reported to induce severe eosinophilic gastroenteritis in a patient with Crohn's Disease ${ }^{11}$. However, we believe, according to our experience that infliximab administration is safe in such patients, and that eosinophilic gastroentritis is more probably to be secondary to the disease instead of the medication.

\section{Conclusion}

In conclusion, the overlap between HES and other conditions, such us in our patient UC, may response to the treatment of the underlying disease ${ }^{12}$. Infliximab can be a good therapeutic alternative, when conventional medical treatment fails. Early diagnosis and treatment of HES have a significant impact on survival that increases from $12 \%$ at 3 years in cases of delayed diagnosis to $80 \%$ at 5 years in the case of early diagnosis ${ }^{1}$.

\section{Consent Statement}

Written informed consent was obtained from the patient for publication of this case report and any accompanying images.

\section{Authors Contribution Section}

MS and PL were in charged of the patient and drafted the manuscript. FG participated in the design of the case report. AF coordinated and helped to draft the manuscript. AL supervised the manuscript once it was finished. All authors read and approved the final manuscript.

\section{References}

1. Jason Gotlib (2014) World Health Organization defined eosinophilic disorders: 2014 update on diagnosis, risk stratification and management. AJH 89(3): 325-337.

2. Simon HU, Rothenberg ME, Bochner BS, Weller PF, Wardlaw AJ, et al. (2010) Refining the definition of hypereosinophilic syndrome. J Allergy Clin Immunol 126(1): 45-49.

3. Valent P, Gleich GJ, Reiter A, Roufosse F, Weller PF, et al. (2012) Pathogenesis and classification of eosinophil disorders: a review of recent developments in the field. Expert Rev Hematol 5(2): 157-176.

4. Pardanani A, Ketterling RP, Brockman SR, Flynn HC, Paternoster SF, et al. (2003) CHIC2 deletion, a surrogate for FIP1L1-PDGFRA fusion, occurs in systemic mastocytosis associated with eosinophilia and predicts response to imatinib mesylate therapy. Blood 102(9): 30933096.

5. Helbig G, Wieczorkiewicz A, Dziacz- kowska-Suszek J, Majewski M, Kyrcz- Krzemien S (2009) T-cell abnormalities are present at high frequencies in patients with hypereosinophilic syndrome. Haematologica 94: $1236-1241$.

6. Mehr S, Rego S, Kakakios A, Kilham H, Kemp A (2009) Treatment of a case of pediatric hypereosinophilic syndrome with anti-interleukin-5. J Pediatr 155(2): 289-291.

7. Verstovsek S, Tefferi A, Kantarjian H, Manshouri T, Luthra R, et al. (2009) Alemtuzumab therapy for hypereosinophilic syndrome and chronic eosinophilic leukemia. Clin Cancer Res 15(1): 368-373.

8. Butterfield JH, Weiler CR (2012) Treatment of hypereosinophilic syndromes the first 100 years. Semin Hematol 49(2): 182-191.

9. Razaq W, Beautyman E (2009) Successful treatment of refractory idiopathic hypereosinophilic syndrome with etoposide. Am J Ther 16(1): 68-70. 
10. Bergua JM, Prieto-Pliego E, Román-Barberá A, García-Torón J, GómezBarrado JJ, et al. (2008) Resolution of left and right ventricular thrombosis secondary to hypereosinophilic syndrome (lymphoproliferative variant) with reduced intensity conditioning allogenic stem cell transplantation. Ann Hematol 87(11): 937-938.

11. Muir A, Surrey L, Kriegermeier A, Shaikhkalil A, Piccoli DA (2016)
Severe Eosinophilic Gastroenteritis in a Crohn's Disease Patient Treated With Infliximab and Adalimumab. Am J Gastroenterol 111(3): 437-438.

12. Terrier B, Fontaine H, Schmitz J, Perdu J, Hermine O, et al. (2007) Coexistence and parallel evolution of hypereosinophilic syndrome autoimmune hepatitis, and ulcerative colitis suggest common pathogenic features. Am J Gastroenterol 102: 1132-1134 